03,08

\title{
Частотная зависимость угла диэлектрических потерь в неупорядоченных полупроводниках в терагерцовой области частот*
}

\author{
() М.А. Ормонт, И.П. Звягин
}

Московский государственный университет им. М.В. Ломоносова, Москва, Россия

E-mail: ormont@phys.msu.ru

\begin{abstract}
Особенности частотной зависимости вещественной части проводимости $\sigma_{1}(\omega)$ в области перехода от почти линейной $(s<1)$ к квадратичной $(s \approx 2)$ могут служить указанием на изменение режима проводимости (переход от переменной к постоянной длине прыжка с ростом частоты); при этом резкость изменения наклона частотной характеристики связана с зависимостью предэкспоненциального множителя резонансного интеграла от межцентрового расстояния в паре. Частотная зависимость мнимой части проводимости $\sigma_{2}(\omega)$ не имеет особенностей в окрестности частоты перехода $\omega_{\mathrm{cr}}$, оставаясь почти линейной. Большая величина котангенса угла диэлектрических потерь $|\operatorname{ctg} \gamma|=\left|\sigma_{2}\right| / \sigma_{1}$ может указывать на то, что при $\omega<\omega_{\text {cr }}$ в области слабого изменения угла потерь $\gamma(\omega)$ мнимая часть проводимости определяется бо́льшим бесфононным вкладом $\sigma_{2}^{\text {res }}$, который существенно превосходит релаксационный $\sigma_{2}^{\text {rel }}$.
\end{abstract}

DOI: 10.21883/FTT.2018.05.45781.08D

\section{1. Введение}

Как известно, исследования диэлектрических потерь (в частности, измерения частотной зависимости проводимости $\left.\sigma(\omega)=\sigma_{1}(\omega)+i \sigma_{2}(\omega)\right)$ позволяют получить информацию о структурных особенностях материала и об особенностях явлений переноса носителей заряда в среде.

Мощность диэлектрических потерь $P$ часто выражают через угол диэлектрических потерь $\gamma$, дополняющий до $\pi / 2$ угол сдвига фаз между током и напряжением; соответственно имеем $\operatorname{tg} \gamma=\sigma_{1} / \sigma_{2}$ и $P=E_{0}^{2} \sigma_{1} / 2=\left(E_{0}^{2} \sigma_{2} / 2\right) \operatorname{tg} \gamma$. Для многих неупорядоченных материалов (аморфные и легированные полупроводники, полупроводниковые стекла, проводящие полимеры, гранулированные проводники и т.п.) частотная зависимость вещественной части проводимости хорошо описывается степенной зависимостью

$$
\sigma_{1}(\omega)=A \omega^{s}
$$

где $A$ и $s-$ постоянные. Как правило, в низкочастотной области показатель степени часто лежит в интервале $0<s<1$ (универсальность) [1], а с ростом частоты в зависимостях $\ln \sigma_{1}(\omega)$ от $\ln \omega$ наблюдается переход от почти линейного $(s \approx 1)$ к квадратичному $(s \approx 2)$ поведению (,излом“) [2-5].

Степенная частотная зависимость (1) указывает на прыжковый характер переноса, однако универсальность зависимости $\sigma(\omega)$ существенно затрудняет получение информации о конкретных особенностях механизма переноса из вида частотной зависимости проводимости.

\footnotetext{
* Доклад на XIV Международной конференции „Физика диэлектриков“" (Санкт-Петербург, 29 мая-2 июня 2017 г.).

Материалы конференции частично опубликованы в выпуске № 3 за 2018 г. журнала „Физика твердого тела“.
}

По этой причине важную роль приобретают исследования отклонений частотной зависимости проводимости от универсальности и установление их связи с особенностями механизма переноса и со структурными особенностями материала.

Существующие теории прыжковой проводимости при переменном токе по локализованным состояниям примесной зоны предсказывают степенные частотные зависимости проводимости (1). В частности, показатель степени $s \approx 1$ получается в случае релаксационной проводимости с участием фононов при условии, что плотность состояний постоянна, а характерная длина прыжка существенно превышает радиус локализованных состояний [6-8]. Близкий к $s \approx 1$ показатель степени получается при низких частотах и в случае бесфононной (резонансной) прыжковой проводимости при учете кулоновских корреляций локализованных носителей [9]. Теория бесфононной проводимости предсказывает переход (кроссовер) от линейной частотной зависимости вещественной части проводимости (с $s \approx 1)$ к зависимости, близкой к квадратичной (с $s \approx 2$ [10]) в области частот порядка $\omega_{\mathrm{cr}}$, при которых $\hbar \omega$ становится порядка энергии кулоновского взаимодействия между электронами внутри резонансных пар; при более низких частотах вещественная часть проводимости определяется фононным механизмом, а с ростом частоты бесфононный вклад в проводимость $\sigma^{\mathrm{res}}(\omega)$ начинает преобладать над релаксационным $\sigma^{\mathrm{rel}}(\omega)$.

Низкотемпературные измерения (при $T \sim 1 \mathrm{~K}$ ) частотной зависимости проводимости в легированном кремнии ( $\mathrm{Si}: \mathrm{B}[2], \mathrm{Si}: \mathrm{P}$ [3-5]) на изоляторной стороне перехода металл-диэлектрик и в металлических нанокомпозитах [11] показали, что с ростом частоты зависимость вещественной части проводимости $\sigma_{1}(\omega)(1)$ переходит от линейной к квадратичной. В Si:P этот переход происходит при частотах порядка $v_{\mathrm{cr}} \sim 1 \mathrm{THz}[3-5]$. 
С учетом кулоновского взаимодействия между электронами на резонансных парах центров выражение для бесфононной проводимости по локализованным состояниям примесной зоны имеет вид [9]

$$
\sigma_{1}^{\mathrm{res}}(\omega)=\frac{1}{3} \pi^{2} e^{2} a g_{\mathrm{F}}^{2} r_{\omega}^{4} \omega\left(\hbar \omega+U\left(r_{\omega}\right)\right),
$$

где $U\left(r_{\omega}\right)=\frac{e^{2}}{\kappa r_{\omega}}-$ энергия кулоновского взаимодействия между электронами на резонансной паре центров, $r_{\omega}=a \ln \left(\omega_{c} / \omega\right)$ - характерная длина прыжка на частоте $\omega, a-$ радиус локализации волновой функции, $\omega_{c}=2 I_{0} / \hbar-$ критическая частота, $I_{0} \sim e^{2} / \kappa a-$ предэкспоненциальный множитель в выражении для резонансного интеграла (порядка энергии ионизации примеси), $\kappa$ - диэлектрическая проницаемость среды, a $g_{\mathrm{F}}$ - плотность локализованных состояний на уровне Ферми.

Частотная зависимость характерной длины прыжка $r_{\omega}$ в режиме резонансной проводимости связана с гибридизацией электронных состояний. Согласно теории $[9,10]$, из-за гибридизации волновых функций изолированной пары центров и соответствующего ей отталкивания уровней, наибольший вклад в бесфононную проводимость вносят пары центров, для которых межцентровые расстояния $r_{\lambda \lambda^{\prime}}$ удовлетворяют неравенствам $r_{\omega} \leq r_{\lambda \lambda^{\prime}} \leq r_{\omega}+a$; при $r_{\lambda \lambda^{\prime}}<r_{\omega}$ отталкивание уровней за счет гибридизации состояний приводит к тому, что $\varepsilon_{\lambda \lambda^{\prime}}^{+}-\varepsilon_{\lambda \lambda^{\prime}}^{-}>\hbar \omega$, и резонансные переходы невозможны. В случае достаточно большого разброса энергий уровней, при условии $\left|\varepsilon_{\lambda^{\prime \prime}}^{0}-\varepsilon_{\lambda}^{0}\right| \gg\left|I_{\lambda, \lambda^{\prime \prime}}\right|$, $\left|\varepsilon_{\lambda^{\prime \prime}}^{0}-\varepsilon_{\lambda^{\prime}}^{0}\right| \gg\left|I_{\lambda^{\prime}, \lambda^{\prime \prime}}\right|$ пары центров $\lambda, \lambda^{\prime}$ с близкими энергиями $\varepsilon_{\lambda}^{0} \approx \varepsilon_{\lambda^{\prime}}^{0}$ можно считать изолированными; при учете гибридизации локализованных электронных состояний в таких парах перекрытием волновых функций центров $\lambda, \lambda^{\prime}$ с другими центрами $\lambda^{\prime \prime}$ можно пренебречь. Характерная длина прыжка $r_{\omega}$ в парном приближении на частоте $\omega$ определяется из условия $\hbar \omega=2 I_{\lambda, \lambda^{\prime}}\left(r_{\omega}\right)$, где $I_{\lambda, \lambda^{\prime}}=I_{0} \exp \left(-r_{\lambda \lambda^{\prime}} / a\right)$ - резонансный интеграл, а $\lambda$ - номер центра. Следует отметить, что характерная длина прыжка $r_{\omega}$ определяется тем, что область значений $\varepsilon_{\lambda \lambda^{\prime}}^{+}-\varepsilon_{\lambda \lambda^{\prime}}^{-}$ограничена снизу величиной $2 I\left(r_{\lambda \lambda^{\prime}}\right)$. Ограничение существенно, когда разность затравочных энергий $\varepsilon_{\lambda^{\prime}}^{0}-\varepsilon_{\lambda}^{0}$ по абсолютной величине меньше $2 I_{\lambda, \lambda^{\prime}}$. $\mathrm{C}$ ростом частоты $\omega$ характерная длина прыжка $r_{\omega}$ уменьшается.

Область применимости теории бесфононной проводимости $[9,10]$ по частоте ограничивается сверху критической частотой $\omega_{c}>\omega$, при которой характерная длина прыжка $r_{\omega}$ становится порядка радиуса локализации состояний (формально при $\omega=\omega_{c}$ характерная длина прыжка $r_{\omega}$ обращается в нуль).

Из выражения (2) видно, что при малых частотах, когда кулоновская энергия превышает энергию кванта электромагнитного поля $\hbar \omega<U\left(r_{\omega}\right)$, для низкотемпературной бесфононной проводимости имеем частотную зависимость типа

$$
\sigma_{1}^{\mathrm{res}}(\omega)=\frac{1}{3} \pi^{2} e^{2} a g_{\mathrm{F}}^{2} r_{\omega}^{4} \omega U\left(r_{\omega}\right)
$$

которую можно аппроксимировать сублинейной степенной функцией $\sigma=A \omega^{s}$ с показателем $s<1$, а при $\hbar \omega>U\left(r_{\omega}\right)$ мы получаем зависимость, близкую к квадратичной,

$$
\sigma_{1}^{\mathrm{res}}(\omega)=\frac{1}{3} \pi^{2} e^{2} a g_{\mathrm{F}}^{2} r_{\omega}^{4} \hbar \omega^{2}
$$

Соотношения (3), (4) описывают линейную и квадратичную частотные зависимости бесфононной прыжковой проводимости (с точностью до логарифмических поправок).

Поскольку $r_{\omega}$ слабо (логарифмически) зависит от частоты, переход (кроссовер) от сублинейной частотной зависимости вещественной части проводимости к квадратичной в (2) происходит при частоте кроссовера $\omega_{\mathrm{cr}}$, определяемой из условия $\hbar \omega_{\mathrm{cr}}=U\left(r_{\omega_{\mathrm{cr}}}\right)$, когда энергия кванта электромагнитного поля $\hbar \omega$ становится равной кулоновской энергии $U\left(r_{\omega}\right)$. Согласно $(2)$, в области кроссовера теория [9] предсказывает плавный переход от линейной частотной зависимости (3) проводимости, связываемой с режимом кулоновского стекла для взаимодействующих электронов, к квадратичной (4) в режиме ферми-стекла для невзаимодействующих электронов.

Сравнение с экспериментом. Одна из трудностей существующей теории [9] состоит в том, что наблюдаемый на опыте переход от линейной к квадратичной частотной зависимости вещественной части проводимости оказывается значительно более резким, чем предсказывает теория [2-5].

В [3] были проведены измерения угла диэлектрических потерь $\gamma$ для легированного кремния $\mathrm{Si}: \mathrm{P}$ в области $\omega<\omega_{\text {cr }}$ и было найдено, что измеренные частотные зависимости вещественной и мнимой частей проводимости близки $\left|\sigma_{2}(\omega)\right| \sim \sigma_{1}(\omega) \sim \omega^{s}(s \approx 1)$. При этом угол потерь $\gamma(\omega)$ слабо зависит от частоты, а частотная зависимость мнимой части проводимости $\sigma_{2}(\omega)$ остается почти линейной. Соответственно, излом на графике зависимости $\ln \left(\sigma_{1}(\omega)\right)$ от $\ln \omega$, отвечающий переходу от линейной к квадратичной частотной зависимости $\sigma_{1}(\omega)$, проявляется и на частотной зависимости величины $\operatorname{ctg} \gamma(\omega)$. Кроме того, оказалось, что в области слабой частотной зависимости $\gamma(\omega)$

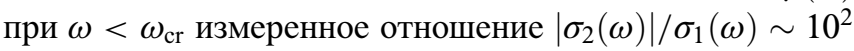
превосходит значение, даваемое теорией релаксационной проводимости, более, чем в 30 раз [12]. Возможная причина этого связана с тем, что проводимость определяется двумя вкладами - резонансным (бесфононным) $\sigma^{\text {res }}(\omega)$ и релаксационным $\sigma^{\text {rel }}(\omega)$; соответственно $\operatorname{ctg}(\gamma)=\left(\sigma_{2}^{\text {res }}+\sigma_{2}^{\text {rel }}\right) /\left(\sigma_{1}^{\text {res }}+\sigma_{1}^{\text {rel }}\right)$. 


\section{2. Влияние гибридизации электронных состояний на высокочастотную проводимость неупорядоченных полупроводников}

Как было отмечено выше, наблюдаемый на опыте переход от линейной к квадратичной частотной зависимости вещественной части проводимости $\sigma_{1}(\omega)$ оказывается существенно более резким, чем предсказывает теория. При этом подход, основанный на парном приближении, предсказывает немонотонность частотной зависимости бесфононной проводимости $\sigma_{1}(\omega)$ и появление плавного максимума на частоте $\omega_{m}=K \omega_{c}$, где $K \approx 0.065$ [13].

Немонотонность частотной зависимости проводимости $\sigma_{1}(\omega)$ определяется двумя конкурирующими тенденциями. Рост $\sigma_{1}(\omega)$ с частотой определяется увеличением энергии поглощаемого кванта $\hbar \omega$ и увеличением числа исходных центров $\sim g\left(\hbar \omega+U\left(r_{\omega}\right)\right)$. Уменьшение $\sigma_{1}(\omega)$ с частотой определяется уменьшением квадрата изменения дипольного момента системы $\left(e r_{\omega}\right)^{2}$ и уменьшением числа конечных центров $\sim g S_{\omega} a$, где $S_{\omega} a=4 \pi r_{\omega}^{2} a-$ объем сферического слоя, в который происходят переходы.

Согласно [13], переход на падающий участок кривой $\sigma_{1}(\omega)$ происходит до того, как достигается область квадратичной зависимости $s \approx 2$. Соответственно вплоть до частоты $\omega_{m}$, отвечающей максимуму $\sigma_{1}(\omega)$, кулоновское взаимодействие между электронами „активных“ пар играет основную роль $U\left(r_{\omega}\right)=e^{2} / \kappa r_{\omega}>\hbar \omega$, и частотная зависимость $\sigma_{1}(\omega)$ остается близкой к линейной $s \approx 1$. Однако экспериментально наблюдаемое поведение проводимости $\sigma_{1}(\omega)$ в области перехода от линейной к квадратичной частотной зависимости не согласуется с предсказываемой теорией немонотонностью [2-5]. Таким образом, в рамках стандартных представлений о переменной длине прыжка $r_{\omega}$, зависящей от частоты, теория резонансной проводимости не описывает наблюдаемый переход от линейной к квадратичной частотной зависимости проводимости $\sigma_{1}(\omega)$.

Наблюдаемый излом на кривых зависимости $\ln \sigma_{1}(\omega)$ от $\ln (\omega)$ может быть связан с переходом к режиму проводимости с постоянной (не зависящей от частоты) длиной прыжка, определяемой параметрами системы [14]. В этом случае оптимальная длина прыжка $r_{\mathrm{opt}}$ перестает зависеть от частоты за счет уменьшения гибридизации электронных состояний: с ростом частоты основной вклад в проводимость начинают вносить пары центров, в которых электрон не успевает гибридизоваться. Гибридизация характеризуется частотой Раби $\omega_{\mathrm{R}}=2 I\left(r_{\lambda \lambda^{\prime}}\right) / \hbar$. При этом для пар с межцентровыми расстояниями $r_{\lambda \lambda^{\prime}} \geq r_{\omega}$ частота Раби меньше частоты поля, т.е. $\omega_{\mathrm{R}}<\omega$, и в этом случае эффекты гибридизации несущественны. При высоких частотах, когда эффекты гибридизации несущественны и оптимальная длина прыжка $r_{\text {opt }}$ не зависит от частоты, выражение для проводимости принимает вид [15]

$$
\sigma_{1}^{\mathrm{res}}(\omega)=\frac{\pi^{2}}{3} C_{1} e^{2} \rho_{0}^{2} a^{5} \omega\left(\hbar \omega+\frac{e^{2}}{\kappa r_{\mathrm{opt}}}\right),
$$

где $C_{1} \approx 10-$ численный множитель, $\rho_{0}-$ одночастичная плотность состояний (считается постоянной). Независящая от частоты характерная длина прыжка $r_{\text {opt }}$ обусловливает монотонный характер частотной зависимости вещественной части проводимости. Существование оптимального расстояния $r_{\text {opt }}$ между центрами в парах обусловлено тем, что с уменьшением расстояния между центрами в паре уменьшается и изменение дипольного момента системы при электронном переходе, а с увеличением расстояния между центрами происходит экспоненциальное уменьшение перекрытия волновых функций состояний, отвечающих центрам локализации.

Согласно (5), переход от линейной частотной зависимости вещественной части проводимости к квадратичной с повышением частоты происходит при частоте $\omega \sim \omega_{\mathrm{cr}}$, где частота кроссовера $\omega_{\mathrm{cr}}$ определяется равенством $\hbar \omega_{\text {cr }}=U\left(r_{\text {opt }}\right)=e^{2} / \kappa r_{\text {opt }}$, т. е. $\omega_{\text {cr }} \sim 0.1 \omega_{c}$; при $a \approx 30 \AA$ частота кроссовера порядка $v_{\mathrm{cr}}=\omega_{\mathrm{cr}} / 2 \pi$ $\sim 1 \mathrm{THz}[15]$.

В некоторых работах (в экспериментах на $\mathrm{Si}: \mathrm{P}[4,5]$ ) наблюдалась суперлинейность $(s>1)$ частотной зависимости проводимости в области частот $\omega<\omega_{\mathrm{cr}}$; это не согласуется с предсказываемой теорией сублинейностью $(s<1)$ (релаксационной [6-8] и бесфононной [9] компонент) в области промежуточных частот. В работах $[4,9]$ суперлинейность частотной зависимости низкотемпературной проводимости неупорядоченных полупроводников в области частот $\omega<\omega_{\mathrm{cr}}$ интерпретировалась как проявление кулоновской щели, возникающей в одночастичной плотности состояний, описывающей распределение самосогласованных энергий взаимодействующих локализованных носителей заряда в основном состоянии системы. В работе [16] отмечалось, однако, что суперлинейность и монотонность экспериментально измеренных частотных зависимостей проводимости $\sigma_{1}(\omega)$ в переходной области частот может быть обусловлена не кулоновской щелью в одночастичной плотности состояний, а постоянной (не зависящей от частоты) оптимальной длиной прыжка и определяющей ролью резонансного механизма проводимости. Оптимальная длина прыжка при этом отвечает переходам вне кулоновской щели.

C ростом частоты характерная длина прыжка $r_{\omega}$ уменьшается, и в области высоких частот происходит переход к режиму проводимости с постоянной длиной прыжка, когда основной вклад в проводимость вносят электронные переходы внутри пар с оптимальными межцентровыми расстояниями $r_{\text {opt}}$, слабо зависящими от частоты. Переход к режиму проводимости с постоянной длиной прыжка $r_{\text {opt }}$ происходит при $r_{\mathrm{opt}} \approx r_{\omega} \quad\left(\right.$ где $\left.r_{\mathrm{opt}} \sim 3 a\right)$, т.е. при частоте $\omega_{\text {opt }}=\exp (-3) \omega_{c} \approx 0.05 \omega_{c}$, и отвечает окрестности частоты кроссовера $\omega_{\mathrm{cr}} \approx 0.1 \omega_{c}$ [14]. Частота $\omega_{\text {opt, }}$ при 


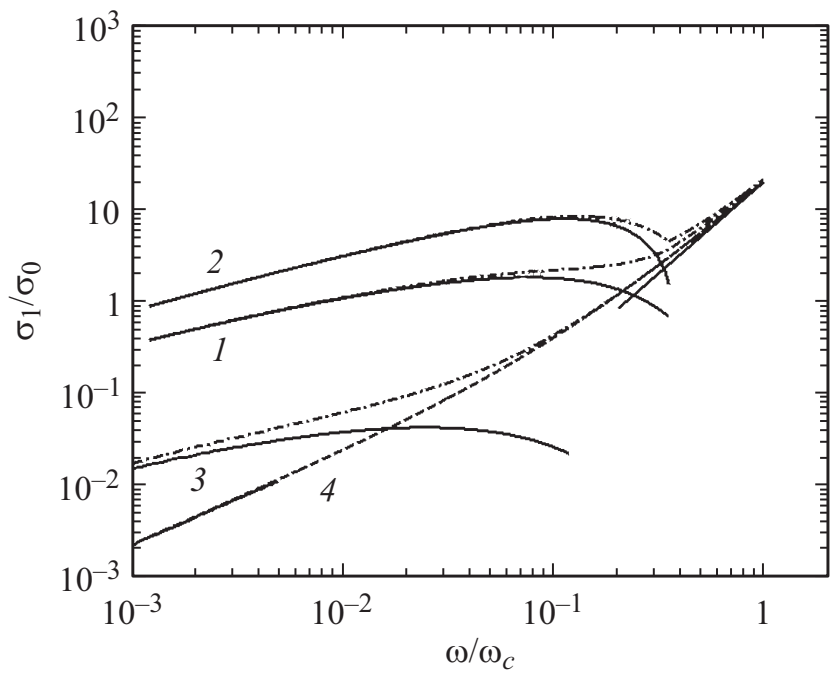

Частотные зависимости вещественной части проводимости $\sigma_{1} / \sigma_{0}$ : кривая $1-$ подход на основе теории бесфононной проводимости в режиме с переменной длиной прыжка $r_{\omega}$ без учета зависимости предэкспоненциального множителя резонансного интеграла от межцентрового расстояния в паре, т.е. с постоянным предэкспоненциальным множителем; кривая 2 - подход на основе теории бесфононной проводимости в режиме с переменной длиной прыжка $\tilde{r}_{\omega}$ с учетом зависимости предэкспоненциального множителя резонансного интеграла от межцентрового расстояния в паре; кривая 3 частотная зависимость релаксационной проводимости (28) с переменной длиной прыжка $\bar{r}_{\omega}$; кривая $4-$ проводимость в режиме с постоянной длиной прыжка; отрезки по краям, обозначенные сплошной линией, приведены для наглядности и соответствуют линейной и квадратичной частотным зависимостям. Штрихпунктирные кривые - экстраполяции моделей с переменной длиной прыжка (соответственно с постоянной предэкспонентой у резонансного интеграла и с предэкспонентой, зависящей от межцентрового расстояния) на область квадратичной частотной зависимости в режиме проводимости с постоянной длиной прыжка (кривая 4). Проводимость приведена в безразмерном виде, где $\sigma_{0}=\frac{\pi^{2} e^{4} \rho_{0}^{2} a^{4} \omega_{c}}{3 \kappa}$.

которой происходит переход к режиму с постоянной длиной прыжка $r_{\text {opt }}$, соответствует положению максимума $\sigma_{1}(\omega)$ в стандартной теории бесфононной проводимости с переменной длиной прыжка. В области частот, где $r_{\text {opt }}>r_{\omega}$, эффекты, связанные с гибридизацией локализованных состояний, становятся несущественны; при этом происходит переход от проводимости в режиме с переменной длиной прыжка к проводимости в режиме с постоянной длиной прыжка. Соответственно, ослабление гибридизации при высоких частотах обусловливает переход от линейной к квадратичной частотной зависимости бесфононной проводимости в терагерцовом диапазоне частот в окрестности $\omega_{\mathrm{cr}}$ (рисунок).

В [17] было исследовано влияние эффектов гибридизации электронных состояний на высокочастотную проводимость неупорядоченных полупроводников, связанное со степенной зависимостью предэкспоненциаль- ного множителя резонансного интеграла. В случае, когда расстояние между центрами в паре больше радиуса локализации, т.е. $r_{\lambda, \lambda^{\prime}}>a$, величины матричных элементов переноса для изотропного закона дисперсии равны

$$
\begin{gathered}
s_{\lambda, \lambda^{\prime}} \approx\left(r_{\lambda, \lambda^{\prime}} / a\right)^{2} \exp \left(-r_{\lambda, \lambda^{\prime}} / a\right), \\
I_{\lambda, \lambda^{\prime}} \approx\left(e^{2} / \kappa a\right)\left(r_{\lambda, \lambda^{\prime}} / a\right) \exp \left(-r_{\lambda, \lambda^{\prime}} / a\right),
\end{gathered}
$$

где $I_{\lambda, \lambda^{\prime}} \approx\left\langle\psi_{\lambda}\left|e^{2} / \kappa\right| \mathbf{r}-\mathbf{r}_{\lambda}|| \psi_{\lambda^{\prime}}\right\rangle-$ резонансный интеграл, $s_{\lambda, \lambda^{\prime}}=\left\langle\psi_{\lambda^{\prime}} \mid \psi_{\lambda}\right\rangle$ - интеграл неортогональности, $\psi_{\lambda}=\left(1 / \sqrt{\pi a^{3}}\right) \exp \left(-\left|\mathbf{r}-\mathbf{r}_{\lambda}\right| / a\right)$ - волновая функция, отвечающая основному состоянию электрона на примеси. Из выражений (6), (7) видно, что предэкспоненциальные множители резонансного интеграла и интеграла неортогональности в случае водородоподобных центров зависят от расстояния между центрами степенным образом. Действительно, при большом межцентровом расстоянии в паре $r_{\lambda, \lambda^{\prime}} \gg a$ основной вклад в резонансный интеграл $I_{\lambda, \lambda^{\prime}}$ и интеграл неортогональности $s_{\lambda, \lambda^{\prime}}$ дает интегрирование по сигарообразной области, вытянутой вдоль отрезка $r_{\lambda, \lambda^{\prime}}$, внутри которой произведение волновых функций $\psi_{\lambda} \psi_{\lambda^{\prime}}$ можно считать постоянным и равным

$$
\psi_{\lambda} \psi_{\lambda^{\prime}} \approx\left(1 / a^{3}\right) \exp \left(-r_{\lambda, \lambda^{\prime}} / a\right)
$$

Степенная зависимость предэкспоненциальных множителей в интегралах $I_{\lambda, \lambda^{\prime}}, s_{\lambda, \lambda^{\prime}}$ от межцентрового расстояния $r_{\lambda, \lambda^{\prime}}$ определяется объемом сигарообразной области, увеличивающимся с ростом межцентрового расстояния. Учет этой зависимости приводит к увеличению длины прыжка $\tilde{r}_{\omega}>r_{\omega}$ и, как следствие, проводимости. С учетом предэкспоненциального множителя длина прыжка $\tilde{r}_{\omega}$ на частоте $\omega$ определяется из условия $\hbar \omega=2 \tilde{I}_{\lambda, \lambda^{\prime}}\left(\tilde{r}_{\omega}\right)$, где $\tilde{I}_{\lambda, \lambda^{\prime}} \approx \frac{e^{2}}{\kappa a} \frac{r_{\lambda, \lambda^{\prime}}}{a} \exp \left(-r_{\lambda, \lambda^{\prime}} / a\right)$. Область частот $\omega<\omega_{c}$ соответствует неравенствам $r_{\omega}, \tilde{r}_{\omega}>a$; при этом с ростом частоты $\omega$ длина прыжка $\tilde{r}_{\omega}$ уменьшается и по мере приближения к частоте кроссовера $\omega_{\text {cr }}$ стремится к величине $r_{\omega}$. Пары с межцентровыми расстояниями $r_{\lambda \lambda^{\prime}}<r_{\omega}, \tilde{r}_{\omega}$ не дают вклада в проводимость, поскольку отталкивание уровней становится бо́льшим $\hbar \omega$ и переходы невозможны. Немонотонность поведения $\sigma_{1}(\omega)$ сохраняется и при учете зависимости предэкспоненциального множителя резонансного интеграла $\tilde{I}_{\lambda, \lambda^{\prime}}$ от межцентрового расстояния в паре (рисунок). Сублинейность $(s<1)$ частотной зависимости $\sigma_{1}(\omega)$ в области $\omega<\omega_{\text {cr }}$ и ее немонотонность обусловлены частотной зависимостью характерных длин прыжка $r_{\omega}, \tilde{r}_{\omega}$.

Учет степенной зависимости предэкспоненциального множителя резонансного интеграла $\tilde{I}_{\lambda, \lambda^{\prime}}$ от расстояния между центрами в паре приводит к существенному уменьшению относительного вклада бесфононной компоненты проводимости в режиме с переменной длиной прыжка в области перехода к режиму проводимости с постоянной длиной прыжка. Такое поведение частотной 
зависимости проводимости $\tilde{\sigma}_{1}^{\mathrm{res}}(\omega)$ обусловлено увеличением длины прыжка $\tilde{r}_{\omega}>r_{\omega}$, и соответственно величины проводимости и связано с быстрым уменьшением длины прыжка $\tilde{r}_{\omega}$ с ростом частоты в области перехода [17].

Таким образом, особенности частотной зависимости проводимости $\sigma_{1}(\omega)$ в области перехода от сублинейности $(s<1)$ к суперлинейности $(s \approx 2)$ могут служить указанием на изменение режима проводимости (переход от переменной к постоянной длине прыжка с ростом частоты); при этом резкость изменения наклона частотной характеристики связана с зависимостью предэкспоненциального множителя резонансного интеграла $\tilde{I}_{\lambda, \lambda^{\prime}}$ от межцентрового расстояния в паре.

В случае мелких примесных уровней (например, для $\mathrm{Si}: \mathrm{P}, \mathrm{Si}: \mathrm{B})$ эффекты гибридизация электронных состояний несущественны в диапазонах $\omega \ll \omega_{\text {cr }}$ и $\omega>\omega_{\text {cr. }}$ В области частот $\omega \ll \omega_{\text {cr }}$ резонансный интеграл мал: $I_{\lambda, \lambda^{\prime}}\left(\bar{r}_{\omega}\right)<k T$, и для характерной длины прыжка при релаксационной проводимости имеем $\bar{r}_{\omega}=(a / 2) \ln \left(\omega_{\mathrm{ph}} / \omega\right)$, где $\omega_{\mathrm{ph}} \sim 10^{12} \mathrm{rad} / \mathrm{s}$. С ростом частоты (т.е. с уменьшением характерных длин прыжка $\left.\bar{r}_{\omega}, r_{\omega}\right)$ резонансная проводимость начинает преобладать над релаксационной, т. е. $\sigma_{1}(\omega) \approx \sigma_{1}^{\text {res }}(\omega)$. В области $\omega>\omega_{\mathrm{cr}}$, отвечающей $r_{\omega}<r_{\mathrm{opt}} \approx 3 a$, происходит уменьшение гибридизации и основной вклад в проводимость вносят переходы внутри пар центров с межцентровыми расстояниями порядка $r_{\text {opt }}$, не зависящими от частоты.

\section{3. Применение соотношений Крамерса-Кронига для анализа электрических свойств неупорядоченных полупроводников}

Как известно, диэлектрическая спектроскопия служит одним из методов получения информации об особенностях механизмов переноса носителей заряда и о локальной структуре материала $[18,19]$. Получение указанной информации связано с исследованием частотных зависимостей функций линейного отклика среды: комплексной диэлектрической восприимчивости $\alpha(\omega)=\alpha_{1}(\omega)+i \alpha_{2}(\omega)$, комплексной проницаемости $\varepsilon(\omega)=\varepsilon_{1}(\omega)+i \varepsilon_{2}(\omega)$ и комплексной проводимости $\sigma(\omega)=\sigma_{1}(\omega)+i \sigma_{2}(\omega)$.

Анализ частотных зависимостей функций $\varepsilon(\omega), \alpha(\omega)$, $\sigma(\omega)$ неупорядоченных систем в изоляторном состоянии $\sigma_{\mathrm{dc}}=0$ часто основывается на соотношениях Крамерса-Кронига (см., например, [3]). Согласно соотношениям Крамерса-Кронига, вещественная и мнимая части функций $\alpha(\omega), \varepsilon(\omega), \sigma(\omega)$ не являются независимыми: спектры вещественной и мнимой частей взаимосвязаны; это дает возможность восстановления функций линейного отклика $\alpha(\omega), \varepsilon(\omega), \sigma(\omega)$ среды по одной из их частей. Дисперсионные соотношения Крамерса-Кронига для комплексной проводимо- сти $\sigma(\omega)$ в отсутствие проводимости на постоянном токе $\sigma_{\mathrm{dc}}=0$ имеют вид

$$
\begin{aligned}
& \sigma_{1}(\omega)=\frac{\omega}{\pi} P \int_{-\infty}^{+\infty} \frac{\sigma_{2}\left(\omega^{\prime}\right)}{\omega^{\prime}\left(\omega^{\prime}-\omega\right)} d \omega^{\prime}, \\
& \sigma_{2}(\omega)=-\frac{\omega}{\pi} P \int_{-\infty}^{+\infty} \frac{\sigma_{1}\left(\omega^{\prime}\right)}{\omega^{\prime}\left(\omega^{\prime}-\omega\right)} d \omega^{\prime} ;
\end{aligned}
$$

где $P \int_{-\infty}^{+\infty}-$ интеграл в смысле главного значения; при этом рассматриваются материалы, не имеющие собственного дипольного момента: $\mathbf{P}(\mathbf{E}=\mathbf{0})=\mathbf{0}$. Соотношения Крамерса-Кронига для диэлектрической проницаемости $\varepsilon(\omega)$ и восприимчивости $\alpha(\omega)$ следуют из (9.1), (9.2), с учетом равенств $\varepsilon=1+4 \pi \alpha$ и $\sigma=-i \omega \alpha$.

Согласно соотношению (9.2), при $0<s<1$ степенной частотной зависимости вещественной части проводимости (1) с постоянным показателем степени соответствует такая же частотная зависимость ее мнимой части [20]

$$
\sigma_{2}(\omega)=-\sigma_{1}(\omega) \operatorname{tg}(\pi s / 2) ;
$$

при этом комплексную проводимость можно представить в виде $\sigma=A_{1}(-i \omega)^{s}$, где $\sigma_{1}=A_{1} \omega^{s} \cos (\pi s / 2)$, $\sigma_{2}=-A_{1} \omega^{s} \sin (\pi s / 2)$. Соотношение (10) было использовано в [3] для анализа поведения комплексной проводимости в области не слишком высоких частот $\omega<\omega_{\mathrm{cr}}$, где $\left|\sigma_{2}(\omega)\right| \sim \sigma_{1}(\omega) \sim \omega^{s}$ с показателем степени $s<1$.

Согласно соотношениям Крамерса-Кронига, вещественная и мнимая части комплексной проводимости $\sigma(\omega)$, так же, как и части комплексной диэлектрической восприимчивости $\alpha(\omega)$, имеют одинаковые частотные зависимости, т. е. их отношение слабо зависит от частоты и определяется показателем степени $s$

$$
\frac{\sigma_{2}(\omega)}{\sigma_{1}(\omega)}=-\frac{\alpha_{1}(\omega)}{\alpha_{2}(\omega)}=-\operatorname{tg}\left(\frac{\pi s}{2}\right)
$$

где

$$
\begin{gathered}
\alpha_{1}(\omega)=-\sigma_{2}(\omega) / \omega=A \omega^{s-1} \operatorname{tg}(\pi s / 2), \\
\alpha_{2}(\omega)=\sigma_{1}(\omega) / \omega=A \omega^{s-1},
\end{gathered}
$$

$\alpha_{1}(\omega), \alpha_{2}(\omega) \sim \omega^{s-1}, \quad \sigma_{1}(\omega), \sigma_{2}(\omega) \sim \omega^{s}, 0<s<1$.

Из (11) с учетом $\operatorname{ctg}(\gamma)=\sigma_{2}(\omega) / \sigma_{1}(\omega)$ следует, что при $s<1$ угол диэлектрических потерь $\gamma$ не зависит от частоты

$$
\operatorname{ctg}(\gamma)=\frac{\sigma_{2}(\omega)}{\sigma_{1}(\omega)}=-\operatorname{tg}\left(\frac{\pi s}{2}\right) .
$$

При значениях $s$, близких к единице, имеем $\frac{\sigma_{2}(\omega)}{\sigma_{1}(\omega)} \approx-\frac{2}{\pi(1-s)},\left|\sigma_{2}\right| / \sigma_{1} \gg 1$, т. е. $\gamma \approx 0$. Согласно $(12)$, случаю малых энергетических потерь $(1 \gtrsim s, \gamma \approx 0$, $\operatorname{tg}(\gamma) \ll 1)$ отвечает слабая частотная зависимость комплексной диэлектрической восприимчивости $\alpha(\omega) \sim \omega^{s-1}$. 
Для частотных зависимостей вещественной и мнимой частей комплексной проводимости теория дает выражения вида $\omega^{m} \ln ^{n}\left(\omega_{c, \mathrm{ph}} / \omega\right)$ (где $n, m$ - целые числа, $\omega_{c, \text { ph }}$ - характерная частота), которые не аппроксимируются степенной функцией с постоянным показателем степени $s$. Характерная фононная частота $\omega_{\mathrm{ph}}$ представляет собой частоту попыток перехода электрона при релаксационной прыжковой проводимости; $\omega_{c}$ есть частота попыток перехода электрона при резонансной проводимости.

\section{4. Расчет частотной зависимости угла потерь неупорядоченных полупроводников в области кроссовера}

Согласно [21], выражение для бесфононной проводимости неупорядоченной изотропной системы имеет вид

$\sigma^{\mathrm{res}}(\omega)=\frac{i e^{2} \omega}{V_{0}} \sum_{\substack{\{i f\} \\ i \neq f}}|\langle i|(\mathbf{n}, \mathbf{r})| f\rangle|^{2} \frac{\left(n_{\mathrm{F}}\left(\varepsilon_{i}\right)-n_{\mathrm{F}}\left(\varepsilon_{f}\right)\right)}{\left(\hbar \omega-\Delta H_{i f}+i \beta\right)}$,

где $i, f$ - номера центров локализации, $V_{0}$ - объем системы, $\mathbf{n}$ - единичный вектор параллельный внешнему электрическому полю, $n_{\mathrm{F}}(\varepsilon)$ - средние числа заполнения состояний с энергией $\varepsilon$;

$$
\Delta H_{i f}=\varepsilon_{f}-\varepsilon_{i}-\frac{e^{2}}{\kappa r_{i f}}=\varphi_{f}-\varphi_{i}
$$

- изменение энергии неупорядоченной системы при одноэлектронном переходе из начального состояния $i$ в конечное состояние $f, r_{i f}$ - расстояние между центрами $i$ и $f, \varphi_{i}, \varphi_{f}$ - энергии электрона в состояниях $i, f$, $\varepsilon_{i}, \varepsilon_{f}$ - самосогласованные энергии электрона, отвечающие состояниям $i, f$ [22]. Выражение (14) получено с использованием адиабатической гипотезы; здесь $\beta-$ малая положительная величина, отвечающая адиабатически медленно возрастающему электрическому полю $\mathbf{E}=\mathbf{E}_{0} \exp \left(-i \omega t+\frac{\beta}{\hbar} t\right)$. Выполнив согласно стандартной процедуре предельный переход $\lim _{\beta \rightarrow 0}$, имеем

$$
\frac{1}{\left(\hbar \omega-\Delta H_{i f}+i \beta\right)}=P \frac{1}{\hbar \omega-\Delta H_{i f}}-i \pi \delta\left(\hbar \omega-\Delta H_{i f}\right),
$$

где $P$ - интеграл в смысле главного значения.

Соответственно вещественная и мнимая части бесфононной проводимости равны

$$
\begin{aligned}
& \sigma_{1}^{\mathrm{res}}(\omega)=\frac{\pi e^{2} \omega}{V_{0}} \\
& \times \sum_{\substack{\{i f\} \\
i \neq f}}|\langle i|(\mathbf{n}, \mathbf{r})| f\rangle|^{2}\left(n_{\mathrm{F}}\left(\varepsilon_{i}\right)-n_{\mathrm{F}}\left(\varepsilon_{f}\right)\right) \delta\left(\Delta H_{i f}-\hbar \omega\right),
\end{aligned}
$$

$$
\sigma_{2}^{\mathrm{res}}(\omega)=\frac{e^{2} \omega}{V_{0}} \sum_{\substack{\{i f\} \\ i \neq f}}|\langle i|(\mathbf{n}, \mathbf{r})| f\rangle|^{2} \frac{\left(n_{\mathrm{F}}\left(\varepsilon_{i}\right)-n_{\mathrm{F}}\left(\varepsilon_{f}\right)\right)}{\left(\hbar \omega-\Delta H_{i f}\right)}
$$

причем при переходе к интегрированию в (17.2) следует рассматривать интеграл в смысле главного значения.

При $r_{i f}>a$ выражение для матричного элемента принимает вид

$$
\langle i|(\mathbf{n}, \mathbf{r})| f\rangle=\left\langle\psi_{i}|(\mathbf{n}, \mathbf{r})| \psi_{f}\right\rangle \approx \frac{r_{i f}^{3}}{a^{2}} \exp \left(-r_{i f} / a\right) \cos \theta,
$$

где $\theta$ - угол между векторами $\mathbf{n}$ и $\mathbf{r}_{i f}$. Матричный элемент имеет максимум при $r_{i f}=3 a$; соответственно основной вклад в мнимую часть проводимости (17.2) вносят слагаемые с $r_{i f} \sim r_{\text {opt }}>a$ и (17.2) можно записать в виде

$$
\sigma_{2}^{\mathrm{res}}(\omega) \approx \frac{e^{2} \omega}{V_{0}} \sum_{\substack{\{i f\} \\ i \neq f}}|\langle i|(\mathbf{n}, \mathbf{r})| f\rangle|^{2} \frac{\left(n_{\mathrm{F}}\left(\varepsilon_{i}\right)-n_{\mathrm{F}}\left(\varepsilon_{f}\right)\right)}{\left(\varepsilon_{i}-\varepsilon_{f}+\frac{e^{2}}{\kappa r_{\mathrm{opt}}}+\hbar \omega\right)} .
$$

Переходя в (19) от суммирования по парам к интегрированию по энергиям и пространственным координатам центров, имеем

$$
\begin{aligned}
& \sigma_{2}^{\mathrm{res}}(\omega) \approx e^{2} \omega \rho_{0}^{2} \\
& \quad \times \iiint d \varepsilon_{i} d \varepsilon_{f} d \mathbf{r}_{i f}|\langle i|(\mathbf{n}, \mathbf{r})| f\rangle|^{2} \frac{\left(n_{\mathrm{F}}\left(\varepsilon_{i}\right)-n_{\mathrm{F}}\left(\varepsilon_{f}\right)\right)}{\left(\varepsilon_{i}-\varepsilon_{f}+\hbar \omega_{1}\right)},
\end{aligned}
$$

где $\hbar \omega_{1} \equiv \frac{e^{2}}{\kappa r_{\mathrm{opt}}}+\hbar \omega$.

Ширину примесной зоны локализованных состояний $\delta$ можно считать существенно бо́льшей ширины кулоновской щели. Напомним, что кулоновская щель, возникающая в одночастичной плотности состояний $\rho(\varepsilon)$, описывающей распределение самосогласованных энергий взаимодействующих локализованных носителей заряда в основном состоянии системы, является следствием дальнодействующего характера кулоновского взаимодействия [23]. Оптимальная длина прыжков, вносящих основной вклад в проводимость, соответствует переходам между состояниями с энергиями, лежащими вне кулоновской щели. Соответственно, в случае столообразной модели плотности состояний кулоновские эффекты, приводящие к появлению кулоновской щели, играют малую роль.

Интегралы по энергии в переходной области частот $\omega \sim \omega_{\mathrm{cr}}$, отвечающей случаю

$$
\hbar \omega_{1}=\frac{e^{2}}{\kappa r_{\mathrm{opt}}}+\hbar \omega<\delta
$$

равны

$$
\iint d \varepsilon_{i} d \varepsilon_{f} \frac{\left(n_{\mathrm{F}}\left(\varepsilon_{i}\right)-n_{\mathrm{F}}\left(\varepsilon_{f}\right)\right)}{\left(\varepsilon_{i}-\varepsilon_{f}+\hbar \omega_{1}\right)} \approx-\delta
$$


при этом мы считаем, что уровень Ферми $\mu$ находится глубоко в примесной зоне.

Подставляя (18) в (20), получаем, что интеграл по пространственным координатам центров в (20) равен

$$
\int d \mathbf{r}|\langle i|(\mathbf{n}, \mathbf{r})| f\rangle|^{2} \approx C_{0} a^{5} \approx r_{\mathrm{opt}}^{5}
$$

где $C_{0}=315$.

Из вида функции (18) следует, что максимум подынтегрального выражения в (24) достигается при значении $r_{i f}=4 a$ и основной вклад в мнимую часть проводимости (17.2) вносят слагаемые с $r_{i f} \sim 4 a \sim r_{\text {орt}}$; при этом (22) не зависит от частоты.

С учетом равенств (21) и (22), в переходной области частот $\omega \sim \omega_{\text {cr }}$, где $\hbar \omega_{1}=\frac{e^{2}}{\kappa r_{\text {opt }}}+\hbar \omega \ll \delta$, выражение для мнимой части бесфононной проводимости (17.2) принимает вид

$$
\sigma_{2}^{\mathrm{res}}(\omega) \approx-e^{2} \omega \rho_{0}^{2} r_{\mathrm{opt}}^{5} \delta
$$

Согласно (23), частотная зависимость мнимой части резонансной проводимости $\sigma_{2}^{\text {res }}$ близка к линейной и имеет емкостной характер (отрицательна).

В режиме с постоянной длиной прыжка, согласно (5), (23), выражение для $\operatorname{ctg}(\gamma)$ имеет вид

$$
\operatorname{ctg}(\gamma)=\frac{\sigma_{2}^{\mathrm{res}}}{\sigma_{1}^{\mathrm{res}}} \approx-10 \frac{\delta}{\hbar \omega_{\mathrm{cr}}} \frac{1}{\left(1+\omega / \omega_{\mathrm{cr}}\right)},
$$

где $\hbar \omega_{\text {cr }}=e^{2} / \kappa r_{\text {opt }} ; \quad$ в области $\omega<\omega_{\text {cr }}$ отношение $\sigma_{2}^{\text {res }} / \sigma_{1}^{\text {res }}$ постоянно и равно $\operatorname{ctg} \gamma \approx-10 \delta / \hbar \omega_{\mathrm{cr}}$.

Согласно (3), (23), с учетом перехода $\sigma_{1}(\omega)$ к режиму проводимости с переменной (зависящей от частоты) длиной прыжка $r_{\omega}$ с понижением частоты в окрестности кроссовера, выражение для $\operatorname{ctg}(\gamma)$ имеет вид

$$
\operatorname{ctg}(\gamma)=\frac{\sigma_{2}^{\mathrm{res}}}{\sigma_{1}^{\mathrm{res}}} \approx-\frac{\delta}{\hbar \omega_{\mathrm{cr}}}\left(\frac{r_{\mathrm{opt}}}{r_{\omega}}\right)^{3} .
$$

Соответственно, в области $\omega<\omega_{\text {cr }}$ вещественная и мнимая части комплексной проводимости имеют близкие частотные зависимости $\left|\sigma_{2}(\omega)\right| \sim \sigma_{1}(\omega) \sim \omega^{s}$ с показателем степени $s \approx 1$; при этом отношение частей проводимости $\left|\sigma_{2}\right| / \sigma_{1}$ слабо зависит от частоты и определяется отношением ширины примесной зоны $\delta$ к характерной кулоновской энергии $\hbar \omega_{\text {cr }} \approx e^{2} / \kappa r_{\text {opt }}, \hbar \omega_{\text {cr }} \ll \delta$.

Примем для оценки разброс уровней, возникающий за счет беспорядка в расположении заряженных примесей, равным $\frac{e^{2}}{\kappa} N_{d}^{1 / 3}$; тогда согласно (24) имеем $\left|\sigma_{2}\right| / \sigma_{1} \approx 10^{2} N_{d}^{1 / 3} a$ Полагая концентрацию примеси $N_{d}=10^{18} \mathrm{~cm}^{-3}$, а радиус локализации $a=30 \AA$, согласно (24), (25), получаем $\left|\sigma_{2}^{\text {res }}\right| / \sigma_{1}^{\text {res }} \sim 10-100$; это по порядку величины согласуется с экспериментально полученными значениями $\left|\sigma_{2}\right| / \sigma_{1}[4]$.

\section{5. Заключение}

Анализ величины и частотной зависимости тангенса угла потерь $\gamma(\omega)$, вообще говоря, требуют одновременного учета как резонансного, так и релаксационного вкладов в проводимость.

Выражения для вещественной и мнимой частей релаксационной проводимости имеют вид

$$
\begin{aligned}
& \sigma_{1}^{\mathrm{rel}}=\frac{\pi^{4} e^{2} \rho_{0}^{2}}{24} a \omega k T \bar{r}_{\omega}^{4}, \\
& \sigma_{2}^{\mathrm{rel}}=-\frac{\pi^{3} e^{2} \rho_{0}^{2}}{30} \omega k T \bar{r}_{\omega}^{5},
\end{aligned}
$$

при $U\left(\bar{r}_{\omega}\right)=\frac{e^{2}}{\kappa \tilde{r}_{\omega}}<k T$ (отметим, что выражение для $\sigma_{1}^{\text {rel }}$ представляет собой соотношение Остина-Мотта [7]). С учетом (26) тангенс угла потерь равен

$$
\operatorname{tg}(\gamma)=\frac{\sigma_{1}^{\mathrm{rel}}}{\sigma_{2}^{\mathrm{rel}}}=-\frac{5 \pi}{2} \ln ^{-1}\left(\frac{\omega_{\mathrm{ph}}}{\omega}\right) .
$$

При низких температурах $U\left(\bar{r}_{\omega}\right)=\frac{e^{2}}{\kappa \tilde{r}_{\omega}} \gg k T$ имеем

$$
\begin{aligned}
& \sigma_{1}^{\mathrm{rel}}=\frac{\pi^{2} e^{4} \rho_{0}^{2}}{6 \kappa} a \omega \bar{r}_{\omega}^{3}, \\
& \sigma_{2}^{\mathrm{rel}}=-\frac{\pi e^{4} \rho_{0}^{2}}{6 \kappa} \omega \bar{r}_{\omega}^{4} ;
\end{aligned}
$$

здесь выражение для $\sigma_{1}^{\text {rel }}$ совпадает с результатом, полученным в [8].

В этом случае тангенс угла потерь с точностью до численного множителя совпадает с (27)

$$
\operatorname{tg}(\gamma)=\frac{\sigma_{1}^{\text {rel }}}{\sigma_{2}^{\text {rel }}}=-2 \pi \ln ^{-1}\left(\frac{\omega_{\mathrm{ph}}}{\omega}\right) .
$$

Как известно, функции вида $\omega^{m} \ln ^{n}\left(\omega_{\mathrm{ph}} / \omega\right)$, фигурирующие в выражениях для проводимости $\sigma_{1}(\omega)$ и $\sigma_{2}(\omega)(26)$, (28), при $\omega \ll \omega_{\text {ph }}$ можно аппроксимировать степенным законом $A \omega^{s}$ с показателем степени

$$
s=\frac{d \ln (\sigma(\omega))}{d \ln \omega}=m-\frac{n}{\ln \left(\omega_{\mathrm{ph}} / \omega\right)} ;
$$

при этом показатель степени $s$ уменьшается с ростом частоты $\omega[20]$.

Согласно (26), (28), вещественная и мнимая части релаксационной проводимости, $\sigma_{1}(\omega)$ и $\sigma_{2}(\omega)$ имеют частотные зависимости $\sigma_{1}(\omega) \sim\left|\sigma_{2}(\omega)\right| \sim \omega^{s}$ с показателем степени $s<1$; при этом для характерных значений параметра $\omega_{\mathrm{ph}} \sim 10^{12}-10^{13} \mathrm{rad} / \mathrm{s}$ в рассматриваемой области частот $v \sim 10^{10}-10^{12} \mathrm{~Hz}$ величина отношения $\left|\sigma_{2}\right| / \sigma_{1}$ порядка единицы. Это не согласуется с аномально большими измеряемыми значениями $|\operatorname{ctg} \gamma|$ в переходной области частот.

Согласно (24), (25), эти расхождения можно объяснить на основе подхода, принимающего во внимание как 
фононный, так и резонансный вклады в проводимость. Бо́льшая величина отношения $|\operatorname{ctg} \gamma|=\left|\sigma_{2}\right| / \sigma_{1}$ указывает на то, что при $\omega<\omega_{\text {cr }}$ в области слабого изменения угла потерь $\gamma(\omega)$ мнимая часть проводимости определяется бо́льшим бесфононным вкладом $\sigma_{2}^{\text {res }}$, который существенно превосходит релаксационный $\sigma_{2}^{\text {rel }}$.

\section{Список литературы}

[1] I.P. Zvyagin. In: Charge Transport in Disordered Solids with Applications in Electronics / Ed. S. Baranovski. John Wiley \& Sons, Chichester (2006). P. 339.

[2] M. Lee, M.L. Stutzmann. Phys. Rev. Lett. 87, 056402 (2001).

[3] E. Helgren, N.P. Armitage, G. Gruner. Phys. Rev. B 69, 014201 (2004).

[4] M. Hering, M. Scheffler, M. Dressel, H.V. Lohneysen. Phys. Rev. B 75, 205203 (2007).

[5] E. Ritz, M. Dressel. Phys. Status Solidi C 5, 703 (2008).

[6] M. Pollak, T.H. Geballe. Phys. Rev. 122, 1742 (1961).

[7] I.G. Austin, N.F. Mott. Adv. Phys. 18, 41 (1969).

[8] A.L. Efros. Philos. Mag. B 43, 829 (1981).

[9] Б.И. Шкловский, А.Л. Эфрос. ЖЭТФ 81, 406 (1981).

[10] N.F. Mott. Philos. Mag. 22, 7 (1970).

[11] J.A. Reedijk, L.J. Adriaanse, H.B. Brom, L.J. de Jongh, G. Schmid. Phys. Rev. B 57, R15116 (1998).

[12] А.Л. Эфрос. ЖЭТФ 89, 1834 (1985).

[13] И.П. Звягин, М.А. Ормонт. Вестн. МГУ. Физика, астрономия, 4, 44 (2008).

[14] М.А. Ормонт, И.П. Звягин. ФТП 49, 4, 449 (2015).

[15] М.А. Ормонт. Вестн. МГУ. Физика, астрономия, 2, 57 (2011).

[16] М.А. Ормонт. ФТП 49, 10, 1314 (2015).

[17] М.А. Ормонт, И.П. Звягин. ФТП 52, 2, 161 (2018).

[18] F. Kremer, A. Schonhals. Broadband dielectric spectroscopy. Springer, Berlin, N. Y. (2003). 729 p.

[19] Г. Фрелих. Теория диэлектриков. Диэлектрическая проницаемость и диэлектрические потери. ИЛ, М. (1960). 251 с.

[20] И.П. Звягин. Кинетические явления в неупорядоченных полупроводниках. Изд-во. МГУ, М. (1984). 192 с.

[21] В.Л. Бонч-Бруевич, И.П. Звягин, Р. Кайпер, А.Г. Миронов, Р. Эндерлайн, Б. Эссер. Электронная теория неупорядоченных полупроводников. Наука, М. (1981). 384 с.

[22] Б.И. Шкловский, А.Л. Эфрос. Электронные свойства легированных полупроводников. Наука, М. (1979). 416 с.

[23] A.L. Efros, B.I. Shklovskii. In: Electron-Electron Interactions in Disordered Systems / Ed. A.L. Efros, M. Pollak. NorthHolland, Elsevier Science Publishers B.V., Amsterdam (1985). P. 409. 